\title{
Magnetic Phase Diagram of Hubbard Model in Three Dimensions: the Second-Order Local Approximation
}

\author{
A.N. Tahvildar-Zadeh ${ }^{1}$, J.K. Freericks ${ }^{2}$ and M. Jarrell ${ }^{1}$ \\ ${ }^{1}$ Department of Physics, University of Cincinnati, Cincinnati, OH 45221 \\ ${ }^{2}$ Department of Physics, Georgetown University, Washington, DC 20057-0995
}

(August 17, 2019)

\begin{abstract}
A local, second-order (truncated) approximation is applied to the Hubbard model in three dimensions. Lowering the temperature, at half-filling, the paramagnetic ground state becomes unstable towards the formation of a commensurate spin-density-wave (SDW) state (antiferromagnetism) and sufficiently far away from half-filling towards the formation of incommensurate SDW states. The incommensurate-ordering wavevector does not deviate much from the commensurate one, which is in accord with the experimental data for the SDW in chromium alloys.
\end{abstract}

Typeset using REVTEX 


\section{INTRODUCTION}

Pure Cr has a spin-density-wave (SDW) ground state with an ordering wavevector which is incommensurate with the underlying latticell. Adding electrons to Cr (by alloying with Mn) makes the magnetic order commensurate with the lattice and increases the transition temperature, whereas removing electrons from the system (by alloying with V) drives the magnetic order to a more incommensurate one, and decreases the transition temperature, eventually to zero (Fig. 5). Penn目 found qualitatively the same behavior for the ground state of a single-band Hubbard model within a mean-field approximation. The mean-field approximation leads to the usual Stoner criterion for the instability of the paramagnetic state to the formation of a SDW state. A first-order perturbation expansion of the self-energy in terms of the interaction parameter leads to the same criterion. In this contribution we extend this approximation one step further to a second-order (non-self-consistent) expansion for the self-energy which includes the lowest-order quantum fluctuations. But, motivated by the case for large spatial dimensions, we ignore the nonlocal (site-non-diagonal) elements

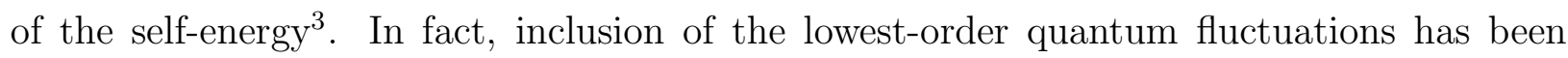
shown to have a dramatic effect on transition temperatures and phase diagrams in the large-dimensional limit, and has been shown to agree with the quantum Monte Carlo data (QMC) over a wide range of interaction strengths 16 .

The Hubbard model is perhaps the simplest model which can be used to study the manybody aspects of correlated electrons on a lattice. The Hubbard Hamiltonian is written in the form

$$
\mathcal{H}=-t \sum_{<i, j>, \sigma}\left(c_{i, \sigma}^{\dagger} c_{j, \sigma}+c_{j, \sigma}^{\dagger} c_{i, \sigma}\right)+U \sum_{i} n_{i, \uparrow} n_{i, \downarrow}
$$

where $c_{i, \sigma}^{\dagger}\left(c_{i, \sigma}\right)$ represents the creation (destruction) operator of an electron in a Wannier

state of spin $\sigma\left(\sigma= \pm \frac{1}{2}\right.$ or $\left.\uparrow \downarrow\right)$, on site $i$, and $n_{i, \sigma}=c_{i, \sigma}^{\dagger} c_{i, \sigma}$ is the electron number operator. The first term corresponds to the kinetic energy and describes the hopping of electrons between nearest-neighbor sites on a lattice via an overlap integral t. This term gives a 
tight-binding description of the electrons in a periodic potential forming a single energy band $\epsilon_{\sigma}(\mathbf{k})=-2 t \sum_{i=1}^{3} \cos k_{i} a$ for a simple cubic lattice with lattice constant $a$. The second term corresponds to the Coulomb repulsion between electrons. The long-range Coulomb interaction is assumed to be screened in the solid so that only the interaction between two electrons on the same site is retained, yielding the additional energy of $U$ when the lattice site is doubly occupied.

The model is specified by three parameters: the strength of the electron interaction $U$ (measured relative to $t$ ); the electron density per spin or electron-filling $n_{e}=\frac{1}{2 N} \sum_{i, \sigma}<$ $c_{i, \sigma}^{\dagger} c_{i, \sigma}>$, where $N$ is the number of sites in the lattice; and the temperature $T$.

In Section II we introduce the formalism and the approximation that we use to form the phase diagram. In Section III, the details of the numerical calculations are described. Section IV, presents the results for the second-order approximation and compares them to the first-order approximation, and the QMC results. A semiquantitative comparison is also made with the experimental data for Cr. Conclusions follow in Section V.

\section{FORMALISM}

For a given $U$ and $n_{e}$ there may exist more than one type of spin order for the ground state, each being stable at a different temperature. Here we start from the paramagnetic state and find a criterion for the instability towards the formation of a SDW state. To do this we couple an external magnetic field to the system and look for singularities in the response function (magnetic susceptibility) as we change the model parameters. The total Hamiltonian of the system in the presence of the magnetic field $h_{i}$ is

$$
\mathcal{H}_{h}=\mathcal{H}-\sum_{i} h_{i} S_{i}^{z} .
$$

where $\mathcal{H}$ is the Hamiltonian in the absence of the external field [found in Eq. (1)] and $i$ is the position of the $i$ th electron with spin $S_{i}^{z}=\sum_{\sigma} \sigma n_{i \sigma}$. The spatial variation of the external field $h_{i}$ is chosen to probe the particular expected order for the spins. For example, if we 
want to examine the instability towards antiferromagnetism we choose $h_{i}$ to be of the same magnitude everywhere but of the opposite sign on the two sublattices of the bipartite lattice.

The static response of the system (or the static spin susceptibility) at temperature $T$ is defined as follows:

$$
\begin{aligned}
\chi_{i j} & =\left.2 \frac{\partial<S_{i}^{z}>}{\partial h_{j}}\right|_{h=0}, \\
& =\left.2 T \sum_{n, \sigma} \sigma \frac{\partial \mathcal{G}_{i i}^{n, \sigma}}{\partial h_{j}}\right|_{h=0} e^{-i \omega_{n} 0^{-}},
\end{aligned}
$$

where $\mathcal{G}_{i j}^{n, \sigma}=\int d \tau e^{-i \omega_{n} \tau}<T_{\tau} c_{j, \sigma}(\tau) c_{i, \sigma}^{\dagger}(0)>$, is Green's function at the Matsubara frequency $\omega_{n}=(2 n+1) \pi T$.

Dyson's equation for Green's function (of the Hamiltonian $\mathcal{H}_{h}$ ) is,

$$
\left(\mathcal{G}^{n, \sigma}\right)_{i j}^{-1}=\left(\mathcal{G}^{0 n, \sigma}\right)_{i j}^{-1}-\Sigma_{i j}^{n, \sigma}+\sigma h_{i} \delta_{i j}
$$

where $\mathcal{G}^{0 n, \sigma}$ is the noninteracting $(U=0)$ Green's function and $\Sigma_{i j}^{n, \sigma}$ is the matrix element of the proper self-energy. Relation (5) and the derivative of the following identity,

$$
\left(\mathcal{G}^{n, \sigma}\right)_{i j}=\left(\mathcal{G}^{n, \sigma}\right)_{i l}\left(\mathcal{G}^{n, \sigma}\right)_{l m}^{-1}\left(\mathcal{G}^{n, \sigma}\right)_{m j}
$$

are employed to find,

$$
\chi_{i j}^{n}=\chi_{i j}^{0 n}+\left.2 \sum_{k, k^{\prime}, l, l^{\prime}} \sum_{n^{\prime}, \sigma, \sigma^{\prime}} \sigma \mathcal{G}_{i k}^{n, \sigma} \mathcal{G}_{l i}^{n, \sigma} \frac{\delta \Sigma_{k l}^{n, \sigma}}{\delta \mathcal{G}_{k^{\prime} l^{\prime}}^{n^{\prime}, \sigma^{\prime}}} \frac{\partial \mathcal{G}_{k^{\prime} l^{\prime}}^{n^{\prime}, \sigma^{\prime}}}{\partial h_{j}}\right|_{h=0},
$$

where $\chi_{i j}=T \sum_{n} \chi_{i j}^{n}$ and the bare susceptibility satisfies $\chi_{i j}^{0 n}=-\sum_{\sigma} \mathcal{G}_{i j}^{n, \sigma} \mathcal{G}_{j i}^{n, \sigma}$.

For the paramagnetic state, we write $\mathcal{G}_{i j}^{n, \sigma}=\mathcal{G}_{i j}^{n,-\sigma}$ and $\frac{\delta \Sigma^{\sigma}}{\delta \mathcal{G}^{\sigma^{\prime}}}=\frac{\delta \Sigma^{-\sigma}}{\delta \mathcal{G}^{-\sigma^{\prime}}}$, so that,

$$
\chi_{i j}^{n}=\chi_{i j}^{0 n}+\left.\sum_{k, k^{\prime}, l, l^{\prime}} \sum_{n^{\prime}} \mathcal{G}_{i k}^{n} \mathcal{G}_{l i}^{n}\left[\frac{\delta \sum_{k l}^{n, \uparrow}}{\delta \mathcal{G}_{k^{\prime} l^{\prime}}^{n^{\prime},}}-\frac{\delta \sum_{k l}^{n, \downarrow}}{\delta \mathcal{G}_{k^{\prime} l^{\prime}}^{n^{\prime}, \uparrow}}\right] \sum_{\sigma^{\prime}} 2 \sigma^{\prime} \frac{\partial \mathcal{G}_{k^{\prime} l^{\prime}}^{n^{\prime}, \sigma^{\prime}}}{\partial h_{j}}\right|_{h=0} .
$$

We need an explicit form for the self-energy to simplify Eq. (8) further. Motivated by the work for large spatial dimension 4 we use a perturbation expansion for the self-energy up to second-order in $U / t$. It was found that the resulting self-energy is almost local in three dimensions $\mathrm{B}$, i.e. $\Sigma_{i j} \approx \Sigma_{i i} \delta_{i j}$, so we employ the local approximation $\Sigma_{i j}=\Sigma_{i i} \delta_{i j}$. 
This approximation becomes exact in large spatial dimensions B $^{3}$, and in three dimensions, the effect of the nonlocal fluctuations on $T_{c}$ is around $3 \%$ in the weak-coupling limit 1 .

Fig. 1 shows the diagrammatic expansion of the local self-energy through second order, which includes the Hartree term and the second-order bubble. Evaluating the diagrams yields,

$$
\Sigma_{i j}^{m, \sigma}=\left[U T \sum_{n} \mathcal{G}_{i i}^{n,-\sigma}-U^{2} T^{2} \sum_{n, n^{\prime}} \mathcal{G}_{i j}^{n, \sigma} \mathcal{G}_{i j}^{n^{\prime},-\sigma} \mathcal{G}_{j i}^{n+n^{\prime}-m,-\sigma}\right] \delta_{i j},
$$

for the second-order local self energy. Substituting this approximation in Eq.(8) and Fourier transforming to the reciprocal lattice, gives a Dyson's-like equation for the susceptibility

$$
\chi(\mathbf{q})=\chi^{0}(\mathbf{q})+T^{2} \sum_{n, n^{\prime}} \chi^{0 n}(\mathbf{q}) \Gamma_{l o c}^{n, n^{\prime}} \chi^{n^{\prime}}(\mathbf{q}),
$$

where $\chi(\mathbf{q})$ is the Fourier transform of $\chi_{i j}$ in the first Brillouin zone and

$$
\Gamma_{l o c}^{n, n^{\prime}}=U\left[1-U \chi_{l o c}^{p p}\left(i \omega_{n+n^{\prime}}\right)\right]
$$

is the irreducible vertex function. $\chi_{l o c}^{p p}$ denotes the local particle-particle susceptibility which is given by,

$$
\chi_{l o c}^{p p}\left(i \omega_{n}\right)=\frac{T}{N^{2}} \sum_{r, \mathbf{p}, \mathbf{p}^{\prime}} \mathcal{G}^{r}(\mathbf{p}) \mathcal{G}^{-r+n}\left(\mathbf{p}^{\prime}\right)
$$

and $\chi^{0}(\mathbf{q})$ is the usual bare particle-hole susceptibility,

$$
\chi^{0}(\mathbf{q})=\frac{-T}{N} \sum_{n, \mathbf{p}} \mathcal{G}^{n}(\mathbf{p}) \mathcal{G}^{n}(\mathbf{p}+\mathbf{q}) .
$$

We expect $\chi\left(\mathbf{q}_{c}\right)$ to diverge at some temperature $T_{c}$ and electron-filling $n_{e c}$ when the system is unstable towards the formation of a SDW at the ordering wavevector $\mathbf{q}_{c}$. So, near the transition temperature, we can neglect $\chi^{0}(\mathbf{q})$ compared to $\chi(\mathbf{q})$ in Eq.(10). At low temperatures, the temperature dependence of $\chi_{l o c}^{p p}$ is found to be negligible and hence $\Gamma^{n, n^{\prime}}$ becomes independent of $n$ and $n^{\prime}$. Thus for low transition temperatures and small $U \chi_{l o c}^{p p}$, we find from Eq.(10) and Eq.(11),

$$
\frac{1}{U}=\chi^{0}\left(\mathbf{q}_{c}, T_{c}, n_{e c}\right)-\chi_{l o c}^{p p}\left(T=0, n_{e c}\right),
$$


as the condition for the transition from the paramagnetic phase to an ordered SDW phase. This equation is called the modified Stoner criterion $\mathrm{B}$ for the magnetic instability of the paramagnetic ground state. If the particle-particle susceptibility is ignored on the righthand-side of Eq. (14), the modified Stoner criterion becomes the usual Stoner criterion; this term results from including the second-order graph in the self-energy expansion.

We must apply one more approximation to calculate the two types of susceptibilities that appear in modified Stoner criterion . We use bare Green's functions in Eq.(12) and Eq.(13) to calculate these susceptibilities. In other words, we are performing a truncated expansion for the self-energy that is not self-consistent. This yields

$$
\chi^{0}(\mathbf{q})=\frac{-T}{(2 \pi)^{3}} \sum_{n} \int d^{3} p \frac{1}{i \omega_{n}-\epsilon(\mathbf{p})+\mu} \cdot \frac{1}{i \omega_{n}-\epsilon(\mathbf{p}+\mathbf{q})+\mu},
$$

and

$$
\chi_{l o c}^{p p}(T=0)=\lim _{T \rightarrow 0} \frac{T}{(2 \pi)^{6}} \sum_{n}\left|\int d^{3} p \frac{1}{i \omega_{n}-\epsilon(\mathbf{p})+\mu}\right|^{2} .
$$

The lowest-order effect of the quantum fluctuations is remarkably simple: just reduce the momentum-dependent particle-hole susceptibility by the local particle-particle susceptibility before applying the Stoner criterion.

\section{NUMERICS}

For each value of $T_{c}, \mathbf{q}_{c}$ and $U$, the root of Eq.(14) yields the critical filling $n_{e c}$ for the SDW order. We use the particle-hole symmetry of the model to find the phase diagram only for values of $n_{e}<0.5$; the phase diagram is symmetric around $n_{e}=0.5$. For a fixed

temperature we can find different roots by changing the ordering wavevector $\mathbf{q}_{c}$. Since the modified Stoner criterion of Eq. (14) is valid only in the nonmagnetic region of the phase diagram, we accept that value of filling for which an instability occurs first, as we approach half-filling, i.e., we search for the $\mathbf{q}_{c}$ in the first Brillouin zone which makes the filling minimal for a fixed temperature. 
Calculations of the modified Stoner criterion for finite-size lattices show that the desired $\mathbf{q}_{c}$ changes only along the edge of the Brillouin zone as the system is doped away from half-filling, i.e. $\mathbf{q}_{c}=\left(\pi, \pi, q_{z}\right)$ for the reduced zone that contains the z-axis. We use this fact to reduce the triple integral in Eq.(15) to an effectively one-dimensional integral in the following way: First note that we can write,

$$
\begin{array}{r}
\chi^{0 n}(\mathbf{q})=\frac{-1}{(2 \pi)^{3}} \int d^{3} p \frac{1}{2\left(i \omega_{n}+\mu\right)+2 t \cos \left(p_{z}\right)+2 t \cos \left(p_{z}+q_{z}\right)} \\
\cdot\left[\frac{1}{i \omega_{n}-\epsilon(\mathbf{p})+\mu}+\frac{1}{i \omega_{n}-\epsilon(\mathbf{p}+\mathbf{q})+\mu}\right] .
\end{array}
$$

This becomes an integral over the $3 \mathrm{D}$ density of states if $q_{z}=\pi$ (i.e. at the zone corner). Note however that the dependence on $p_{x}$ and $p_{y}$ is only through $-2 t \cos \left(p_{x}\right)-2 t \cos \left(p_{y}\right)$ which allows the $p_{x}$ and $p_{y}$ integrals to be replaced by an integral over the $2 \mathrm{D}$ density of states, and produces local Green's functions in 2D:

$$
\begin{aligned}
\chi^{0 n}(\mathbf{q})=\frac{-1}{2 \pi} \int d p_{z} \frac{1}{2\left(i \omega_{n}+\mu\right)+2 t \cos \left(p_{z}\right)+2 t \cos \left(p_{z}+q_{z}\right)} \\
.\left[\mathcal{G}_{2 D}\left(i \omega_{n}+2 t \cos \left(p_{z}\right)+\mu\right)+\mathcal{G}_{2 D}\left(i \omega_{n}+2 t \cos \left(p_{z}+q_{z}\right)+\mu\right)\right] .
\end{aligned}
$$

Here $\mathcal{G}_{2 D}(z)$ is the local 2D Green's function,

$$
\mathcal{G}_{2 D}(z)=\int d \epsilon \frac{\rho_{2 D}(\epsilon)}{z-\epsilon}
$$

$\mathcal{G}_{2 D}(z)$ is evaluated with a quadrature algorithm which employs a rational function expansion for large $|z|$, and employs a 512 point Gaussian integration when $|z|$ is small. So we can evaluate $\chi^{0}(\mathbf{q})$ efficiently by numerically performing the remaining integration over $p_{z}$. Note that $\chi_{l o c}^{p p}$ is already in the form of a one-dimensional integral over the 3D density of states.

\section{RESULTS}

Fig. 2 shows the resulting phase boundary for the Hubbard model using the modified Stoner criterion. At half-filling the transition is always commensurate with the lattice, i.e. 
$\mathbf{q}=\frac{\pi}{a}(1,1,1)$. As the system is doped away from half-filling the transition temperature decreases and a point is reached where the transition becomes incommensurate with the underlying lattice. Finite-size calculations show that the ordering wavevector changes only along the Brillouin zone edge as the transition becomes incommensurate, i.e. $\mathbf{q}=\frac{\pi}{a}(1,1,1-$ $\delta)$. The inset in Fig. 2 shows that $\delta$ remains small and eventually stops changing as the filling is changed. Note that the ordering wavevector initially changes very rapidly at the incommensurate order onset, which is reminiscent of the first-order jump seen in the $\mathrm{Cr}$ datal.

Fig. 3 shows the result of a finite-size calculation for the original Stoner criterion which results from a first-order (Hartree) approximation for the self-energy. We see that the transition temperature is enhanced by almost a factor of four for each value of $U$ compared to the second-order approximation results at half-filling. For a given value of $U$ the change in slope at the onset of incommensuration is much smaller than the results of the second-order approximation. This change of slope in the second-order approximation is again similar to what is seen in the Cr data. Also, as was already shown by Penn, in the first-order approximation, for values of $U$ large enough, the ordering wavevector changes first along the edge of the zone, but then continues to move toward the zone center as the system is doped far away from half-filling. This latter behavior has suggested that the system can become ferromagnetic for low electron filling which is ruled out once the quantum fluctuations are included.

Fig. 4 shows a comparison of the transition temperature versus $U$ at half-filling for the first and the second-order approximations as well as the result of a quantum Monte Carlo (QMC) simulation?. We see that in the case of the second-order approximation, $T_{c}$ has negative curvature for $U / t$ around 10 similar to the QMC result (although the former is not a valid approximation for large values of $U$ ), whereas in the first-order approximation $T_{c}$ continues to increase with $U$ with a positive curvature. In fact, since the second-order approximation is exact as $U \rightarrow 0$, it is clear that the QMC data is overestimating $T_{c}$ by a factor of four in the weak-coupling limit. Such criticism has already been raised by comparing 
to other approximation methods 11 .

Electronic band structure calculations 1 show that the $d$-electron concentration for pure $\mathrm{Cr}$ is 2.28 electrons/atom/spin. In order to map this onto our single-band model, we assume that $1 / 5$ of this contributes to our single-band filling, implying $n_{e}=0.456$ (there are five d-bands in $\mathrm{Cr}$ ). The Fermi energy of pure $\mathrm{Cr}$ is near the edge of a $d$-band so that the density of states has a large peak there, thus we assume that doping with Mn adds one electron to the single band, and doping with $\mathrm{V}$ removes one electron from it. This maps the rest of the experimental data to the single-band model. We then fit the value of $U$ in the modified Stoner criterion, to reproduce the experimental filling for the onset of incommensurate order. As shown in Fig. 5, the fit is remarkably good for $U=5.5 t$. Note that there is only one adjustable parameter in this fit. The agreement is remarkable since the lattice structure for $\mathrm{Cr}$ is actually bcc and because we made such simple assumptions. Furthermore, the experimental shape cannot be properly accounted for with a first-order approximation for any reasonable value of $U$.

\section{CONCLUSIONS}

We found a criterion for the instability of the paramagnetic ground state of the 3D Hubbard model towards the formation of an ordered SDW state. This criterion is a simple modification of the Stoner criterion and is derived by including the second-order graph in the expansion of the local self-energy. Hence this new criterion includes the lowest-order corrections of the old Stoner criterion due to quantum fluctuations.

We showed that the magnetic phase boundary between paramagnetic and SDW phases

of the model changes both quantitatively and qualitatively when we include the effect of quantum fluctuations to the usual mean-field (Hartree) result. The quantum fluctuations suppress the transition temperatures and exclude the possibility of the formation of ferromagnetism in the 3D Hubbard model.

We showed that the resulting phase boundary from this modified Stoner criterion is 
consistent with the experimental data for the SDW in dilute chromium alloys whereas there are features in the data that cannot be accounted for by the usual Stoner criterion with reasonable values of the interaction strength. We were indeed able to achieve remarkable agreement with the experimental data, although this model is too simple to be a realistic one for Cr. However, since the modified Stoner criterion is a simple and efficient approximation, we hope it can be applied to more realistic models for $\mathrm{Cr}$ and other transition metals.

\section{ACKNOWLEDGMENTS}

We would like to acknowledge useful conversations with R. Fishman and P. van Dongen. This work was supported at the University of Cincinnati by the National Science Foundation Grant No. DMR-9406678 and DMR-9357199. J. K. F. acknowledges the Donors of The Petroleum Research Fund, administered by the American Chemical Society, for partial support of this research (ACS-PRF\# 29623-GB6). 


\section{REFERENCES}

${ }^{1}$ E. Fawcett, Rev. Mod. Phys. 60, 209 (1988) and references therein.

${ }^{2}$ D. R. Penn, Phys. Rev. 142, 350 (1966).

${ }^{3}$ W. Metzner, D. Vollhardt, Phys. Rev. Lett. 62, 324 (1989).

${ }^{4}$ P. G. J. van Dongen, Phys. Rev. Lett. 67, 757 (1991).

${ }^{5}$ M. Jarrell, Phys. Rev. Lett. 69, 168 (1992).

${ }^{6}$ J. K. Freericks and M. Jarrell, Phys. Rev. Lett. 74, 186 (1995).

${ }^{7}$ J. Hubbard, Proc. R. Soc. a276, 238 (1963)

${ }^{8}$ H. Schweitzer, G. Czycholl, Z. Phys. B 83, 93 (1991).

${ }^{9}$ R. T. Scalettar, D. J. Scalapino, R. L. Sugar, D. Toussaint, Phys. Rev. B 39, 4711 (1989).

${ }^{10}$ Y. Kakehashi, H. Hasegawa, Phys. Rev. B 37, 7777 (1988). 


\section{FIGURES}

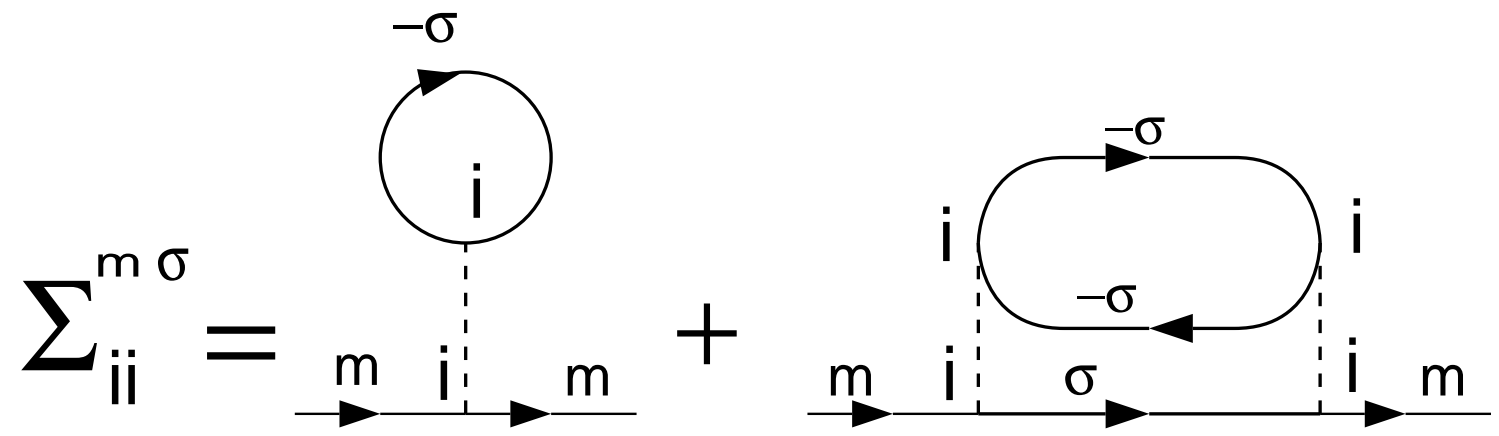

FIG. 1. The local self-energy $\Sigma_{i i}$ in the second-order approximation to the Hubbard model. The Fock term is absent in the Hubbard model. The solid line represents the undressed $(U=0)$ electron Green's function $\mathcal{G}_{i j}^{0}\left(i \omega_{n}\right)$ and the dotted line represents the intrasite interaction $U$. The external legs just show the Matsubara frequency dependence and are not included in the analytic expressions. 


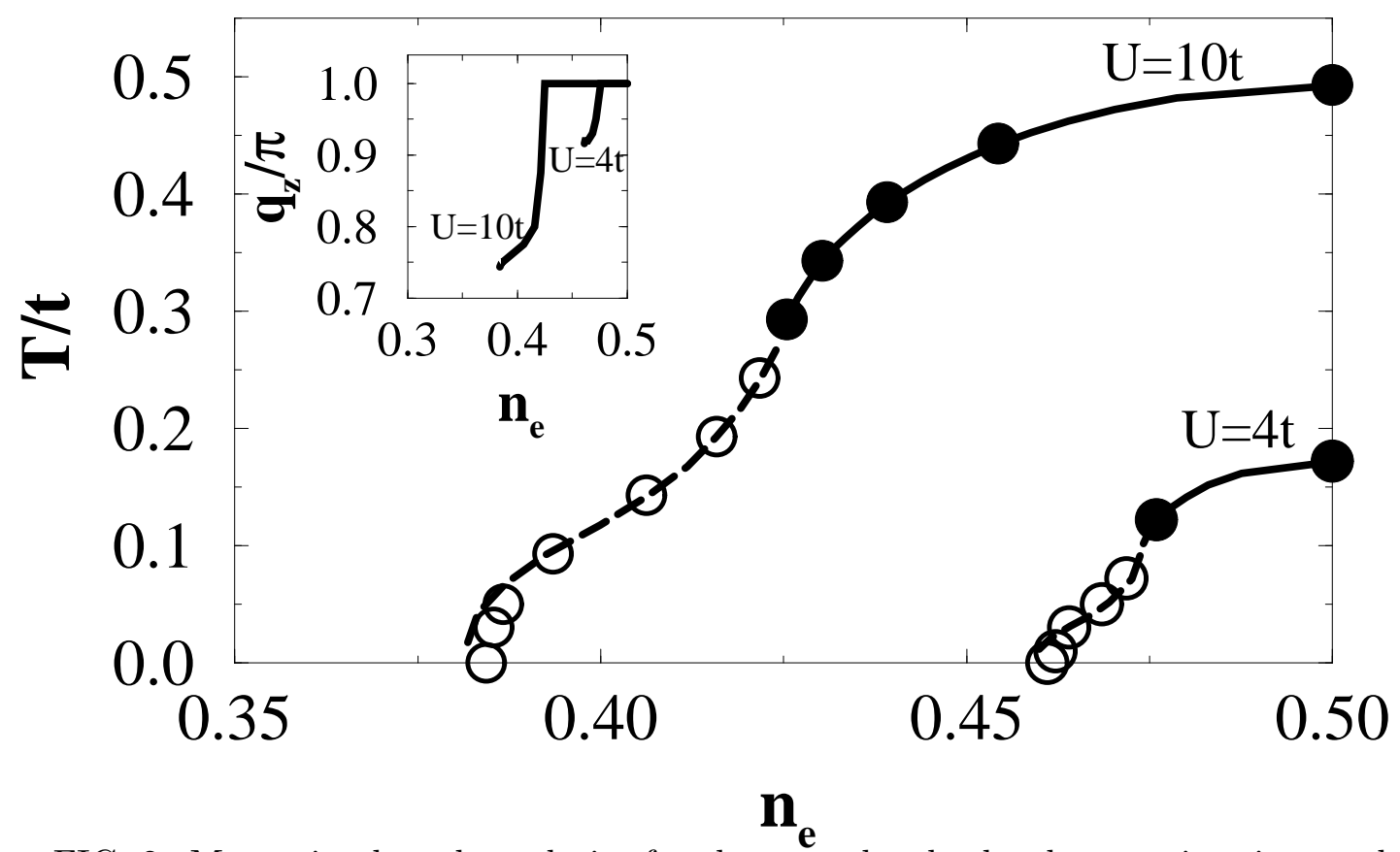

FIG. 2. Magnetic phase boundaries for the second-order local approximation to the Hubbard model in three dimensions for two different values of the interaction strength $U$. The vertical axis shows the ratio of the temperature to the hopping constant. The horizontal axis shows the electron filling. The (dashed) solid lines denote the (in)commensurate transition from a paramagnetic to a spin-density-wave ground state in the thermodynamic limit. The (open) solid circles denote the corresponding transitions for a finite lattice of 80 unit cells for $T>0.05 t$ and 500 unit cells for lower temperatures. The inset shows the corresponding z-component of the magnetic ordering wavevector $\mathbf{q}$ versus filling. The phase-diagram is symmetric around $n_{e}=0.5$. 


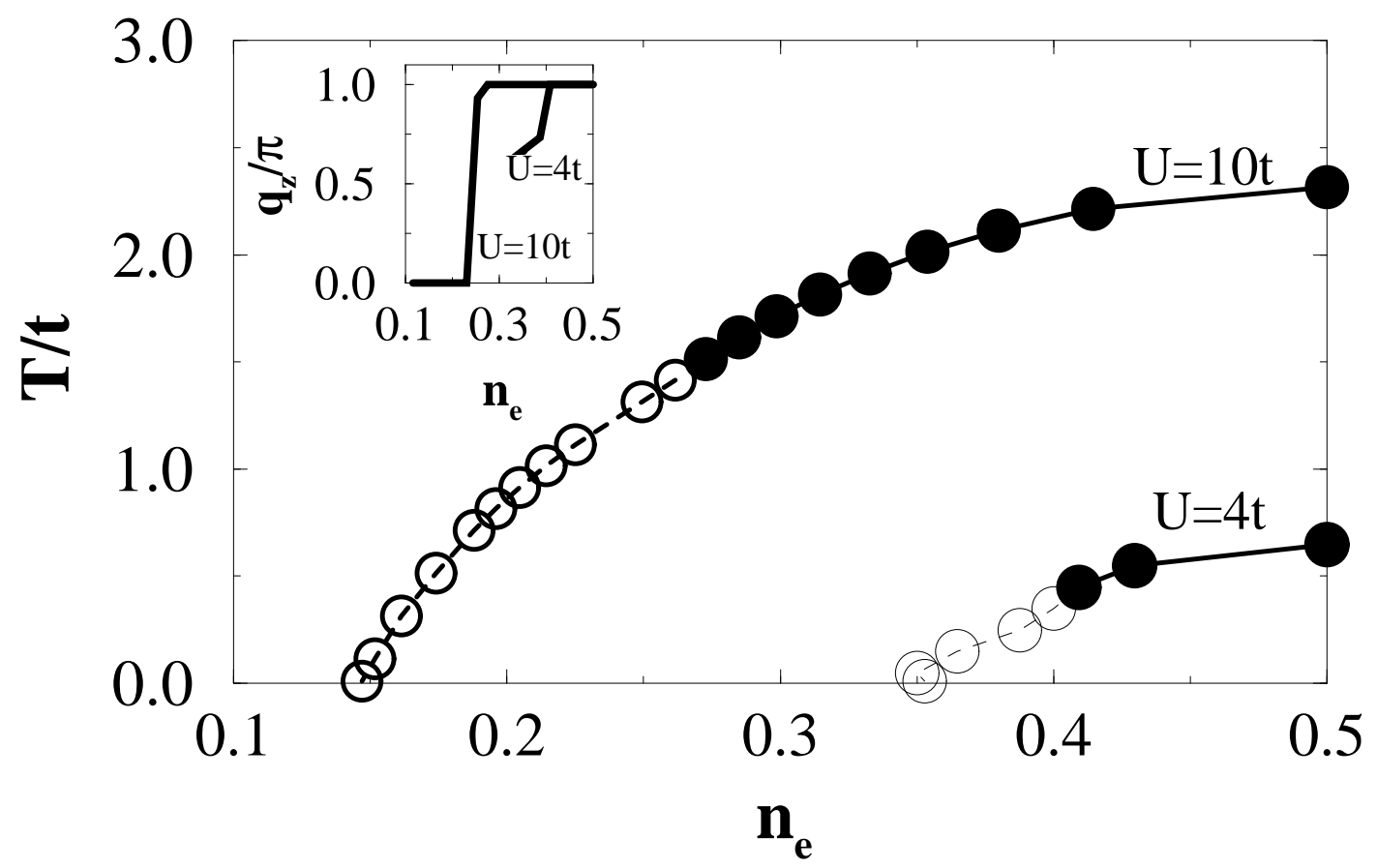

FIG. 3. Magnetic phase boundaries for the first-order approximation to the Hubbard model in three dimensions for two different values of the interaction strength $U$. The vertical axis shows the ratio of the temperature to the hopping constant. The horizontal axis shows the electron filling. The (open) solid circles denote (in) commensurate transition from a paramagnetic to a spin-density-wave ground state for a finite lattice of 30 unit cells. The lines are a guide to the eye. The inset shows the corresponding z-component of the magnetic ordering wavevector q versus filling. The phase-diagram is symmetric around $n_{e}=0.5$. 


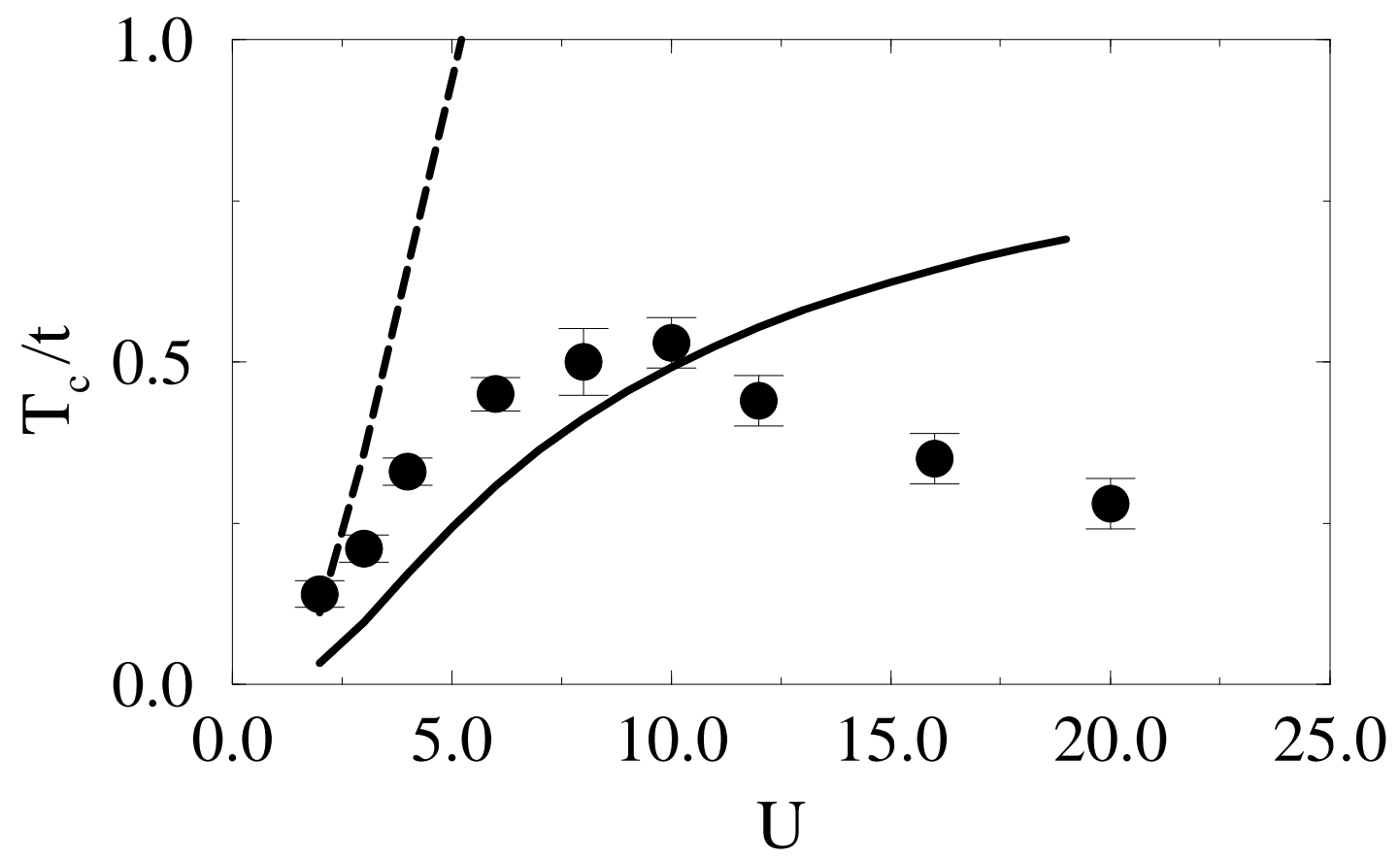

FIG. 4. Comparison between the results of quantum Monte Carlo simulations ${ }^{9}$ (circles), the first-order (dashed line) and the second-order local approximation (solid line). $T_{c} / t$ is the ratio of the transition temperature at half-filling to the hopping integral. $U$ is the interaction strength. The second-order local approximation shows the same sign of the curvature as the QMC result, whereas the first-order curve shows the wrong sign of the curvature. Also, since the second-order approximation has the correct limiting behavior as $U \rightarrow 0$, this shows that the QMC data is overestimating $T_{c}$ for small values of $U$. 


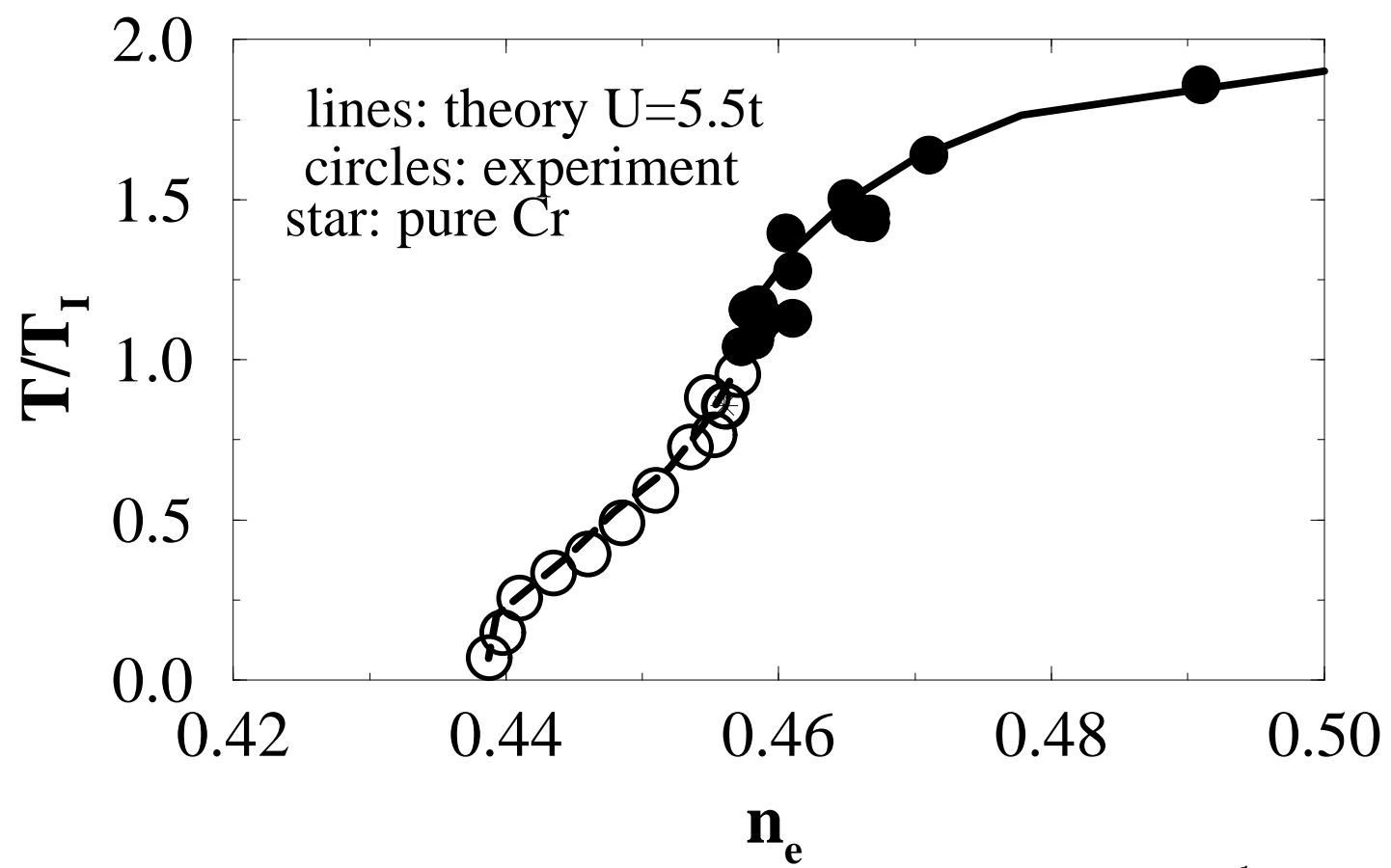

FIG. 5. Comparison between the experimental data for chromium alloys ${ }^{1}$ and the results of the second-order local approximation. The (open) closed circles denote the experimental data for the (in)commensurate transitions in Cr alloys. The (dashed) solid lines denote the results of the second-order local approximation for the (in)commensurate transitions. The experimental data is best fit to the theoretical calculation when $U=5.5 t . T_{I}$ is the transition temperature at the paramagnetic-commensurate-incommensurate phase boundary. $T_{I}=360 \mathrm{~K}$ for experimental data and occurs when $\mathrm{Cr}$ is doped with 0.3 atomic percent of Mn. 